\title{
Estimation of the mean of functional time series and a two sample problem
}

\author{
Lajos Horváth \\ Piotr Kokoszka* \\ University of Utah, Salt Lake City, USA Utah State University, Logan, USA \\ Ron Reeder \\ University of Utah, Salt Lake City, USA
}

\begin{abstract}
This paper is concerned with inference based on the mean function of a functional time series, which is defined as a collection of curves obtained by splitting a continuous time record, e.g. into daily or annual curves. We develop a normal approximation for the functional sample mean, and then focus on the estimation of the asymptotic variance kernel. Using these results, we develop and asymptotically justify a testing procedure for the equality of means in two functional samples exhibiting temporal dependence. Evaluated by means of a simulations study and application to real data sets, this two sample procedure enjoys good size and power in finite samples. We provide the details of its numerical implementation.
\end{abstract}

Keywords: Functional data analysis; Time series; Two sample problem; Long-run variance; Eurodollar futures.

Abbreviated Title: Functional time series

* Address for correspondence: Piotr Kokoszka, Department of Mathematics and Statistics, Utah State University, 3900 Old Main Hill, Logan, UT 84322-3900, USA. E-mail: Piotr.Kokoszka@usu.edu 


\section{Introduction}

Functional time series form a class of data structures which occurs in many applications, but several important aspects of estimation and testing for such data have not received as much attention as for functional data derived from randomized experiments. In the latter case, the curves can often be assumed to form a simple random sample, in particular, the functional observations are independent. For curves obtained from splitting a continuous (in principle) time records into, say, daily or annual curves, the assumption of independence is often violated. This paper focuses on the methodology and theory for the estimation of the mean function of a functional time series, and on inference for the mean of two functional time series. Despite their central importance, these issues have not yet been studied. The contribution of this paper is thus two-fold: 1) we develop a methodology and an asymptotic theory for the estimation of the variance of the sample mean of temporally dependent curves under model-free assumptions; 2) we propose procedures for testing equality of two mean functions in functional samples exhibiting temporal dependence.

A functional time series $\left\{X_{k}, k \in \mathbb{Z}\right\}$ is a sequence of curves $X_{k}(t), t \in[a, b]$. After normalizing to the unit interval, the curves are typically defined as $X_{k}(t)=X(k+t), 0 \leq$ $t \leq 1$, where $\{X(u), u \in \mathbb{R}\}$ is a continuous time record, which is often observed at equispaced dense discrete points. An example is given in Figure 1.1, which shows seven consecutive functional observations. More examples are studied in Horváth and Kokoszka (2011). A central issue in the analysis of such data is to take into account the temporal dependence of the observations. The monograph of Bosq (2000) studies the theory of linear functional time series, focusing on the functional autoregressive model. For many functional time series it is however not clear what specific model they follow, and for many statistical procedures it is not necessary to assume a specific model. In this paper, we assume that the functional time series is stationary, but we do not impose any specific model on it. We assume that the curves are dependent in a very broad sense, which is made precise in Section 1.1. The dependence condition we use is however satisfied by all models for functional time series used to date, including the linear, multiplicative, bilinear and ARCH type processes. We refer to Hörmann and Kokoszka (2010) and ? for examples.

A direct motivation for the research presented in this paper comes from a two sample problem in which we wish to tests if the mean functions of two functional time series are equal. A specific problem, studied in greater depth in Section 4, is to test if the mean curves of certain financial assets are equal over certain periods. This in turn allows us to conclude whether the expectations of future market conditions are the same or different at specific time periods. In general, if the same mean is assumed for the whole time series, whereas, in fact, it is different for disjoint segments, the inference or exploratory analysis 
FIGURE 1.1. The horizontal component of the magnetic field measured in one minute resolution at Honolulu magnetic observatory from 1/1/2001 00:00 UT to 1/7/2001 24:00 UT.

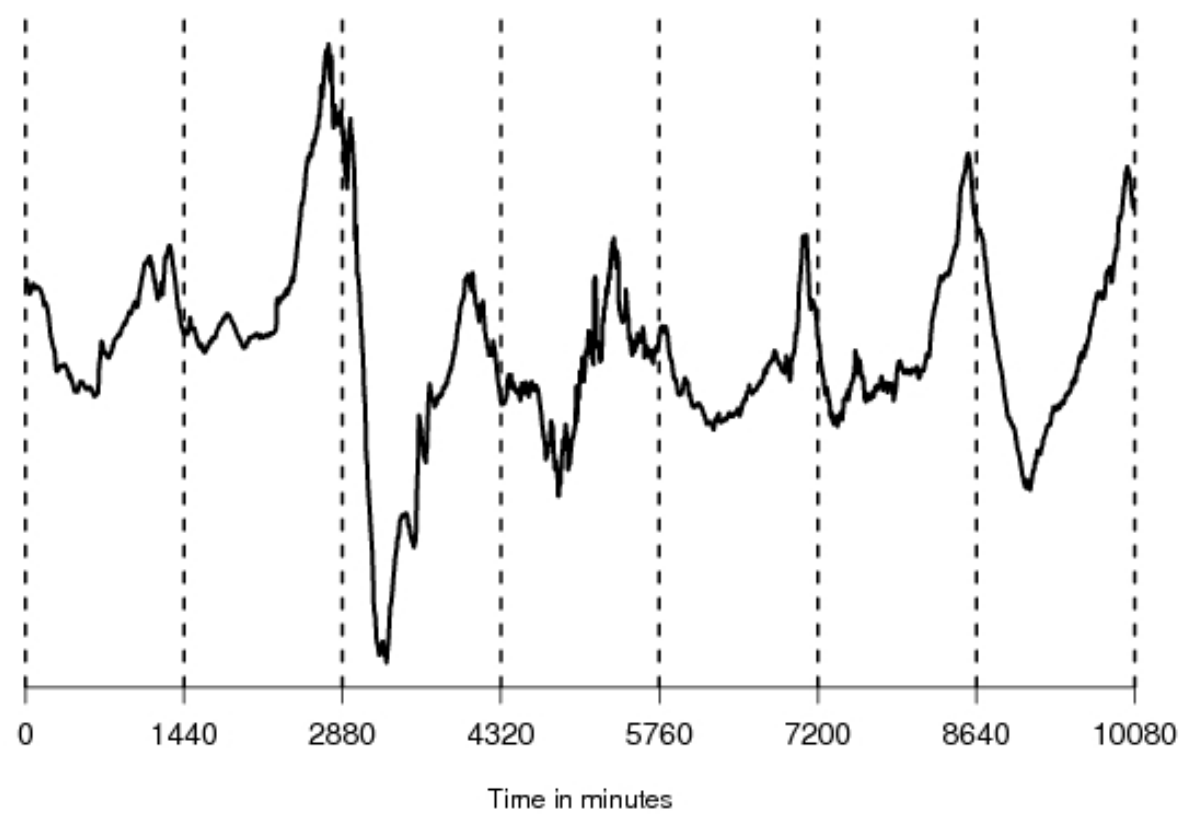

that follows will be faulty, as all prediction and model fitting procedures for functional time series start with subtracting the sample mean, viewed as an estimate of the unique population mean function. The same holds true for independent curves; if two subsamples have different mean functions, subtracting the sample mean function based on the whole data set will lead to spurious results. Despite the importance of two sample problems for functional data, they have received little attention. Recent papers of Horváth et al. (2009) and Panaretos et al. (2010) are the only contributions to a two sample problem in a functional setting which develop inferential methodology. Horváth et al. (2009) compare linear operators in two functional regression models. Panaretos et al. (2010) focus on testing the equality of the covariance operators in two samples of iid Gaussian functional observations; our paper focuses on the means of dependent (and non-Gaussian) observations. We develop the required methodology, justify it by asymptotic arguments, and describe its practical implementation.

Any inference involving mean functions requires estimates of the variability of the sample mean. In iid functional samples, the sample covariance operator is used, but for functional time series this problem is much more difficult. For scalar and vector-valued time series, the variance of the sample mean is asymptotically approximated by the long-run 
variance whose estimation has been one of the central problems of time series analysis, studied in textbooks, see e.g. Anderson (1971), Brockwell and Davis (1991), Hamilton (1994), and dozens of influential papers, see ?, Andrews (1991) and Andrews and Monahan (1992), to name just a few. Convergence of various estimators of the long-run variance has been established under several types of assumptions, including broad model specifications (e.g. linear processes), cumulant conditions, and various mixing conditions. Hörmann and Kokoszka (2010) and Gabrys et al. (2010) advocated using the notion of $L^{p}-m$-approximability for functional time series, as this condition is intuitively appealing and is easy to verify for functional time series models. We therefore develop a general framework for the estimation of the long-run covariance kernel in this setting. The longrun covariance kernel (equivalently operator) is an infinite dimensional object, precise definitions are given in Section 2, whose estimation has not been studied yet. Hörmann and Kokoszka (2010) studied the estimation of a long-run covariance matrix obtained by projecting this operator onto a finite dimensional basis. Their approach is based on the results for vector-valued time series, and they use cumulant-like assumptions which are difficult to verify for nonlinear functional time series.

The long-run covariance kernel corresponds to the asymptotic variance in a normal approximation for the sample mean of a scalar time series, but no central limit theorem for a general functional time series has been established yet (results for linear processes are established in Bosq (2000)). We provide such a generally applicable result as well (Theorem 2.1).

The remainder of the paper is organized as follows. We conclude the introduction by defining the notion of dependence for functional time series which we use throughout this paper. Then, in Section 2, we state the asymptotic results for the mean of a single functional time series, with proofs developed in Section 5 . Section 3 focuses on the problem of testing the equality of means of two functional samples which exhibit temporal dependence. In Section 4, we evaluate the finite sample performance of the procedures proposed in Section 3 by means of a simulation study and application to real data. The proofs of the theorems stated in Section 3 are collected in Section 6 .

\subsection{Approximable functional time series}

We consider a stationary functional time series $\left\{\varepsilon_{i}(t), i \in \mathbb{Z}, t \in[0,1]\right\}$, which we can view as an error sequence in a more complex functional model, for example a regression model, as in Gabrys et al. (2010). We assume that these errors are nonlinear moving averages $\varepsilon_{i}=f\left(\delta_{i}, \delta_{i-1}, \ldots\right)$, for some measurable function $f: S^{\infty} \rightarrow L^{2}$, and iid elements $\delta_{i}$ of a measurable space $S$. In all models used in practice $S=L^{2}$. To motivate the 
construction below, it is useful to write the $\varepsilon_{i}$ as

$$
\varepsilon_{i}=f\left(\delta_{i}, \ldots, \delta_{i-m+1}, \delta_{i-m}, \delta_{i-m-1} \ldots\right)
$$

Under (1.1), the sequence $\left\{\varepsilon_{i}\right\}$ is stationary and ergodic. The function $f$ must decay sufficiently fast to ensure that the sequence $\left\{\varepsilon_{i}\right\}$ is weakly dependent. The weak dependence condition is stated in terms of an approximation by $m$-dependent sequences, namely, we require that

$$
\sum_{m \geq 1}\left[E \int\left(\varepsilon_{i}(t)-\varepsilon_{i, m}(t)\right)^{2} d t\right]^{1 / 2}<\infty
$$

where

$$
\varepsilon_{i, m}=f\left(\delta_{i}, \ldots, \delta_{i-m+1}, \delta_{i, i-m}^{(m)}, \delta_{i, i-m-1}^{(m)}, \ldots\right),
$$

with the sequences $\left\{\delta_{i, k}^{(m)}\right\}$ being independent copies of the sequence $\left\{\delta_{i}\right\}$. Note that the sum in 1.2 does not depend on $i$.

The idea behind the above construction is that the function $f$ decays so fast that the effect of the innovations $\delta_{i}$ far back in the past becomes negligible; they can be replaced by different, fully independent innovations. If the $\varepsilon_{i}$ follow a linear model $\varepsilon_{i}=\sum_{j \geq 0} c_{j}\left(\delta_{i-j}\right)$, condition (1.2) intuitively means that the approximations by the finite moving averages $\varepsilon_{i, m}=\sum_{0 \leq j \leq m} c_{j}\left(\delta_{i-j}\right)$ become increasingly precise. This means that the operators $c_{j}$ must decay sufficiently fast in an appropriate operator norm. We refer to Hörmann and Kokoszka (2010) and ? for the details and examples of nonlinear functional time series satisfying 1.2 .

We also note that the general idea of using nonlinear moving averages (Bernoulli shifts) and imposing moment conditions to quantify dependence has been recently used in other contexts, see $\mathrm{Wu}(2005,2007)$. The connections between such notions and the traditional mixing conditions, or other notions of weak dependence, e.g. that introduced by Doukhan and Louhichi (1999), are only partially understood at present. In particular, it is not clear which functional time series models satisfy dependence conditions other than the approximability 1.2 .

\section{Normal approximation and long-run variance for func- tional time series}

In this section, we state the central limit theorem for the sample mean of an $L^{2}-m-$ approximable functional time series. Its applicability depends on the estimation of the 
covariance kernel of the limit. We therefore also establish the consistency of the kernel estimator of the long-run covariance kernel. We assume that $\left\{\varepsilon_{i}\right\}$ is an $L^{2}-m$-approximable (and hence stationary) functional time series satisfying

$$
E \varepsilon_{0}=0, \text { in } L^{2}
$$

and

$$
\int E \varepsilon_{0}^{2}(t) d t<\infty
$$

Theorem 2.1. If (1.1), (1.2), (2.1), (2.2) hold, then

$$
N^{-1 / 2} \sum_{i=1}^{N} \varepsilon_{i} \stackrel{d}{\rightarrow} Z \quad \text { in } \quad L^{2},
$$

where $Z$ is a Gaussian process with

$$
\begin{gathered}
E Z(t)=0 \quad \text { and } E[Z(t) Z(s)]=c(t, s) \\
c(t, s)=E \varepsilon_{0}(t) \varepsilon_{0}(s)+\sum_{i \geq 1} E \varepsilon_{0}(t) \varepsilon_{i}(s)+\sum_{i \geq 1} E \varepsilon_{0}(s) \varepsilon_{i}(t) .
\end{gathered}
$$

The infinite sums in the definition of the kernel c converge in $L^{2}([0,1] \times[0,1])$, i.e. c is a square integrable function on the unit square.

Theorem 2.1 is proven in Section 5 .

The kernel $c$ is defined analogously to the long-run variance of a scalar time series. It is directly related to the covariance operator of the sample mean defined by

$$
\begin{aligned}
\hat{C}_{N}(x) & =N E\left[\left\langle\frac{1}{N} \sum_{i=1}^{N} \varepsilon_{i}, x\right\rangle \frac{1}{N} \sum_{j=1}^{N} \varepsilon_{j}\right] \\
& =\frac{1}{N} \sum_{i, j=1}^{N} E\left[\left\langle\varepsilon_{i}, x\right\rangle \varepsilon_{j}\right] .
\end{aligned}
$$

If the $\varepsilon_{i}$ are independent, then $\hat{C}_{N}(x)=N^{-1} \sum_{i=1}^{N} E\left[\left\langle\varepsilon_{i}, x\right\rangle \varepsilon_{i}\right]$ becomes the usual sample (empirical) covariance operator, which plays a central role in many exploratory and inferential tools of functional data analysis of iid functional observations, mostly through the empirical functional principal components defined as its eigenfunctions. For functional time series, it is not suitable. For any stationary functional time series $\left\{\varepsilon_{i}\right\}$,

$$
\begin{aligned}
\hat{C}_{N}(x)(t) & =\int\left(\frac{1}{N} \sum_{i, j=1}^{N} E\left[\varepsilon_{i}(s) \varepsilon_{j}(t)\right]\right) x(s) d s \\
& =\int c_{N}(t, s) x(s) d s,
\end{aligned}
$$


where

$$
c_{N}(t, s)=\sum_{|k|<N}\left(1-\frac{|k|}{N}\right) E\left[\varepsilon_{0}(s) \varepsilon_{k}(t)\right] .
$$

The summands in 2.5 converge to those in (2.4), but the estimation of the long-run covariance kernel $c$ is far from trivial.

To enhance the applicability of our result, we state it for the case of a nonzero mean function, which is estimated by the sample mean. We thus assume that

$$
X_{i}(t)=\mu(t)+\varepsilon_{i}(t), \quad 1 \leq i \leq N,
$$

with the series $\left\{\varepsilon_{i}\right\}$ satisfying the assumptions of Theorem 2.1.

Let $K$ be a kernel (weight) function defined on the line and satisfying the following conditions:

$$
\begin{gathered}
K(0)=1, \\
K \text { is continuous, } \\
K \text { is bounded, } \\
K(u)=0, \text { if }|u|>c, \text { for some } c>0 .
\end{gathered}
$$

Condition 2.10 is assumed only to simplify the proofs, a sufficiently fast decay could be assumed instead.

Next we define the empirical (sample) correlation functions

$$
\hat{\gamma}_{i}(t, s)=\frac{1}{N} \sum_{j=i+1}^{N}\left(X_{j}(t)-\bar{X}_{N}(t)\right)\left(X_{j-i}(s)-\bar{X}_{N}(s)\right), \quad 0 \leq i \leq N-1
$$

where

$$
\bar{X}_{N}(t)=\frac{1}{N} \sum_{1 \leq i \leq N} X_{i}(t)
$$

The estimator for $c$ is given by

$$
\hat{c}_{N}(t, s)=\hat{\gamma}_{0}(t, s)+\sum_{i=1}^{N-1} K\left(\frac{i}{h}\right)\left(\hat{\gamma}_{i}(t, s)+\hat{\gamma}_{i}(s, t)\right)
$$

where $h=h(N)$ is the smoothing bandwidth satisfying

$$
h(N) \rightarrow \infty, \quad \frac{h(N)}{N} \rightarrow 0, \quad \text { as } N \rightarrow \infty .
$$


In addition to $(1.2)$, we also assume that

$$
\lim _{m \rightarrow \infty} m\left[E \int\left(\varepsilon_{n}(t)-\varepsilon_{n, m}(t)\right)^{2} d t\right]^{1 / 2}=0 .
$$

Theorem 2.2. Suppose the functional time series $\left\{X_{i}\right\}$ follows model (2.6). Under conditions (1.1), (1.2), (2.1), (2.2), (2.7)-(2.10), (2.13), (2.14),

$$
\iint\left(\hat{c}_{N}(t, s)-c(t, s)\right)^{2} d t d s \stackrel{P}{\rightarrow} 0
$$

with $c(t, s)$ by defined by (2.4) and $\hat{c}_{N}(t, s)$ by (2.12).

Theorem 2.2 is proven in Section 5. First we use the results of this section in the problem of testing the equality of means in two functional samples.

\section{Testing the equality of mean functions}

We consider two samples of curves, $X_{1}, X_{2}, \ldots, X_{N}$ and $X_{1}^{*}, X_{2}^{*}, \ldots X_{M}^{*}$, satisfying the following location models

$$
X_{i}(t)=\mu(t)+\varepsilon_{i}(t), \quad X_{j}^{*}(t)=\mu^{*}(t)+\varepsilon_{j}^{*}(t) .
$$

The error functions $\varepsilon_{i}$ are assumed to satisfy the conditions stated in Sections 1 and 2 . The functions $\varepsilon_{j}^{*}$ are assumed to satisfy exactly the same conditions. In particular, their long-run covariance kernel is defined by

$$
c^{*}(t, s)=E \varepsilon_{0}^{*}(t) \varepsilon_{0}^{*}(s)+\sum_{i \geq 1} E \varepsilon_{0}^{*}(t) \varepsilon_{i}^{*}(s)+\sum_{i \geq 1} E \varepsilon_{0}^{*}(s) \varepsilon_{i}^{*}(t) .
$$

We assume that

$$
\left\{\varepsilon_{i}, 1 \leq i \leq N\right\} \text { and }\left\{\varepsilon_{j}^{*}, 1 \leq j \leq M\right\} \quad \text { are independent. }
$$

We are interested in testing

$$
H_{0}: \mu=\mu^{*}
$$

against the alternative

$$
H_{A}: \mu \neq \mu^{*}
$$

The equality in 3.3 is in the space $L^{2}=L^{2}([0,1])$, i.e. $\mu=\mu^{*}$ means that $\int\left(\mu(t)-\mu^{*}(t)\right)^{2} d t=$ 0 , and the alternative means that $\int\left(\mu(t)-\mu^{*}(t)\right)^{2} d t>0$. 
Since the statistical inference is about the mean functions of the observations, our procedures are based on the sample mean curves

$$
\bar{X}_{N}(t)=\frac{1}{N} \sum_{1 \leq i \leq N} X_{i}(t) \quad \text { and } \quad \bar{X}_{M}^{*}=\frac{1}{M} \sum_{1 \leq i \leq M} X_{j}(t) .
$$

The sample means $\bar{X}_{N}$ and $\bar{X}_{M}^{*}$ are unbiased estimators of $\mu$ and $\mu^{*}$, respectively, so $H_{0}$ will be rejected if

$$
U_{N, M}=\frac{N M}{N+M} \int\left(\bar{X}_{N}(t)-\bar{X}_{M}^{*}(t)\right)^{2} d t
$$

is large.

Before introducing the test procedures, we state two results which describe the asymptotic behavior of the statistic $U_{N, M}$ under $H_{0}$ and $H_{A}$. They motivate and explain the development that follows.

Theorem 3.1. Suppose $H_{0}$, the assumptions of Theorem 2.1 (and analogous assumptions for the $\varepsilon_{j}^{*}$ ) and (3.2) hold. If

$$
\frac{N}{N+M} \rightarrow \theta, \quad \text { for some } 0 \leq \theta \leq 1, \quad \text { as } \min (M, N) \rightarrow \infty,
$$

then

$$
U_{N, M} \stackrel{d}{\rightarrow} \int_{0}^{1} \Gamma^{2}(t) d t
$$

where $\{\Gamma(t), 0 \leq t \leq 1\}$ is a mean zero Gaussian process with covariances

$$
E[\Gamma(t) \Gamma(s)]=d(t, s):=(1-\theta) c(t, s)+\theta c^{*}(t, s) .
$$

Theorem 3.2. If $H_{A}$, and the remaining assumptions of Theorem 3.1 hold, then

$$
\frac{N+M}{N M} U_{N, M} \stackrel{P}{\rightarrow} \int_{0}^{1}\left(\mu(t)-\mu^{*}(t)\right)^{2} d t .
$$

In particular, if $0<\theta<1$, then $U_{N, M} \stackrel{P}{\rightarrow} \infty$.

The kernel $d(t, s)$ in Theorem 3.1 defines a covariance operator $D$. The eigenvalues of $D$ are nonnegative, and are denoted by $\lambda_{1} \geq \lambda_{2} \geq \ldots$. By the Karhunen-Loéve expansion, we have

$$
\int_{0}^{1} \Gamma^{2}(t) d t=\sum_{i=1}^{\infty} \lambda_{i} N_{i}^{2}
$$

where $\left\{N_{i}, 1 \leq i<\infty\right\}$ are independent standard normal random variables. 
Since the eigenvalues $\lambda_{i}$ are unknown, the right-hand side of (3.6) cannot be used directly to simulate the distribution of $\int \Gamma^{2}(t) d t$. We will now explain how to estimate the $\lambda_{i}$ 's. Suppose $\hat{D}_{N, M}$ is an $L^{2}$-consistent estimator of $D$, i.e.

$$
\iint\left(\hat{d}_{N, M}(t, s)-d(t, s)\right)^{2} d t d s \stackrel{P}{\rightarrow} 0, \quad \text { as } \min (M, N) \rightarrow \infty .
$$

We discuss the construction of estimators $\hat{D}_{N, M}$ satisfying 3.7 below. For the estimators we propose, relation (3.7) holds regardless whether $H_{0}$ or $H_{A}$ holds, they do not depend on $\mu$ or $\mu^{*}$ either. We will also see that the critical relations 6.1 hold under $H_{A}$ as well as under $H_{0}$. The distribution of $\int \Gamma^{2}(t) d t$ can thus be estimated also under the alternative. Let

$$
\hat{\lambda}_{1}=\hat{\lambda}_{1}(N, M) \geq \hat{\lambda}_{2}=\hat{\lambda}_{2}(N, M) \geq \ldots
$$

denote the eigenvalues of $\hat{D}_{N, M}$, i.e.

$$
\int \hat{d}_{N, M}(t, s) \hat{\varphi}_{i}(s) d s=\hat{\lambda}_{i} \hat{\varphi}_{i}(t)
$$

where the $\hat{\varphi}_{i}(t)=\hat{\varphi}_{i}(t ; N, M)$ are the corresponding eigenfunctions satisfying $\int \hat{\varphi}_{i}^{2}(t) d t=$ 1. Choosing $p$ so large that $\sum_{i=1}^{p} \hat{\lambda}_{i}$ is a large percentage of $\sum_{i=1}^{N+M} \hat{\lambda}_{i}$, we can approximate the distribution of $\int \Gamma^{2}(t) d t$ by that of $\sum_{i=1}^{p} \hat{\lambda}_{i} N_{i}^{2}$.

The statistical inference is based on the difference $\bar{X}_{N}-\bar{X}_{M}^{*}$. Observe that

$$
\frac{M N}{M+N} E\left[\left(\bar{X}_{N}(t)-\bar{X}_{M}^{*}(t)\right)\left(\bar{X}_{N}(s)-\bar{X}_{M}^{*}(s)\right)\right] \rightarrow d(t, s), \quad \text { as } \min (M, N) \rightarrow \infty,
$$

that is, $d$ is the asymptotic covariance kernel of the difference $\bar{X}_{N}-\bar{X}_{M}^{*}$. We therefore use projections onto the eigenfunctions $\varphi_{1}, \varphi_{2}, \ldots, \varphi_{p}$ associated with the $p$ largest eigenvalues of $D$. This is analogous to projecting onto the functional principal components in one sample problems, as these form an $L^{2}$-optimal orthonormal basis. Without any loss of generality, we assume that the $\varphi_{1}, \varphi_{2}, \ldots, \varphi_{p}$ form an orthonormal system (the $\varphi_{i}$ are orthogonal under (3.13), so only a normalization to unit norm is required). We define the projections

$$
a_{i}=\left\langle\bar{X}_{N}-\bar{X}_{M}^{*}, \varphi_{i}\right\rangle, \quad 1 \leq i \leq p
$$

and the vectors

$$
\mathbf{a}=\left[a_{1}, a_{2}, \ldots, a_{p}\right]^{T} .
$$

We show in the proof of Theorem 3.3 that

$$
\left(\frac{M N}{M+N}\right)^{1 / 2} \mathbf{a} \stackrel{d}{\rightarrow} \mathbf{N}_{p}(\mathbf{0}, \mathbf{Q})
$$


where $\mathbf{N}_{p}(\mathbf{0}, \mathbf{Q})$ stands for the $p$-variate normal random vector with mean zero and the covariance matrix $\mathbf{Q}=\operatorname{diag}\left(\lambda_{1}, \lambda_{2}, \ldots, \lambda_{p}\right)$. Since the operator $D$ is unknown, we cannot compute the $\varphi_{i}$. However, any estimator for $D$ satisfying (3.7) can be used to find estimates for the $\varphi_{i}$. Let $\hat{\varphi}_{i}$ be the empirical eigenfunctions defined by (3.8), and set

$$
\hat{a}_{i}=\left\langle\bar{X}_{N}-\bar{X}_{M}^{*}, \hat{\varphi}_{i}\right\rangle, \quad 1 \leq i \leq p
$$

The limit relation 3.10 suggests the following statistics:

$$
U_{N, M}^{(1)}=\frac{M N}{M+N} \sum_{i=1}^{p} \hat{a}_{i}^{2}
$$

and

$$
U_{N, M}^{(2)}=\frac{M N}{M+N} \sum_{i=1}^{p} \frac{\hat{a}_{i}^{2}}{\hat{\lambda}_{i}} .
$$

The following theorem establishes the limits of $U_{N, M}^{(1)}$ and $U_{N, M}^{(1)}$ under $H_{0}$.

Theorem 3.3. Suppose $H_{0}$, the remaining assumptions of Theorem 3.1. (3.7) and

$$
\lambda_{1}>\lambda_{2}>\ldots>\lambda_{p}>\lambda_{p+1}
$$

hold. Then

$$
U_{N, M}^{(1)} \stackrel{d}{\rightarrow} \sum_{i=1}^{p} \lambda_{i} N_{i}^{2},
$$

where $N_{1}, N_{2}, \ldots, N_{p}$ are independent standard normal random variables, and

$$
U_{N, M}^{(2)} \stackrel{d}{\rightarrow} \chi^{2}(p)
$$

where $\chi^{2}(p)$ stands for a chi-square random variable with $p$ degrees of freedom.

We note that $U_{N, M}^{(1)}$ is essentially the first $p$ terms in the Karhunen-Loéve expansion of the integral in the definition of $U_{N, M}$. Thus, the limit in (3.14) is exactly the random variable we used to approximate the distribution of $U_{N, M}$. The limit in $(3.15)$ is distribution free. The consistency of the tests based on $U_{N, M}^{(1)}$ and $U_{N, M}^{(2)}$ follows from the following result.

Theorem 3.4. Suppose $H_{A}$, the remaining assumptions of Theorem 3.1. (3.7) and (3.13) hold. Then

$$
\frac{N+M}{N M} U_{N, M}^{(1)} \stackrel{P}{\rightarrow} \sum_{i=1}^{p}\left\langle\mu-\mu^{*}, \varphi_{i}\right\rangle^{2}
$$


and

$$
\frac{N+M}{N M} U_{N, M}^{(2)} \stackrel{P}{\rightarrow} \sum_{i=1}^{p} \frac{\left\langle\mu-\mu^{*}, \varphi_{i}\right\rangle^{2}}{\lambda_{i}} .
$$

In particular, if $0<\theta<1$ in $\sqrt{3.5)}$, then $U_{N, M}^{(1)} \stackrel{P}{\rightarrow} \infty$ and $U_{N, M}^{(2)} \stackrel{P}{\rightarrow} \infty$, provided $\left\langle\mu-\mu^{*}, \varphi_{i}\right\rangle \neq 0$ for at least one $1 \leq i \leq p$.

We see that the condition for the consistency is that $\mu-\mu^{*}$ is not orthogonal to the linear subspace of $L^{2}$ spanned by the eigenfunctions $\varphi_{i}, 1 \leq i \leq p$.

The implementation of the tests based on Theorems 3.3 and 3.4 depends on the existence of an estimator of the kernel $d(t, s)$ which satisfies (3.7). The remainder of this section is dedicated to this issue.

The estimation of $D$ is very simple if the $\varepsilon_{i}$ are iid, and the $\varepsilon_{j}^{*}$ are iid. In this case, setting,

$$
\hat{\theta}=\frac{N}{N+M},
$$

we can use

$$
\hat{d}_{N, M}(t, s)=(1-\hat{\theta}) \hat{c}_{N}(t, s)+\hat{\theta} \hat{c}_{M}^{*}(t, s),
$$

where

$$
\begin{gathered}
\hat{c}_{N}(t, s)=\frac{1}{N} \sum_{i=1}^{N}\left(X_{i}(t)-\bar{X}_{N}(t)\right)\left(X_{i}(s)-\bar{X}_{N}(s)\right) ; \\
\hat{c}_{M}^{*}(t, s)=\frac{1}{M} \sum_{j=1}^{M}\left(X_{j}^{*}(t)-\bar{X}_{M}^{*}(t)\right)\left(X_{j}^{*}(s)-\bar{X}_{N}^{*}(s)\right) .
\end{gathered}
$$

By condition (2.2), we can use the weak law of large numbers in a Hilbert space to establish (3.7). The estimation of $D$ is much more difficult if only $(1.2)$ is assumed, and its asymptotic justification relies on Theorem 2.2. Recall the definition of the estimator $\hat{c}_{N}(t, s)$ given in (2.12), and define the estimator $\hat{c}_{M}^{*}(t, s)$ fully analogously. Our estimator for $d(t, s)$ is then (3.16) with $\hat{c}_{N}(t, s)$ and $\hat{c}_{M}^{*}(t, s)$ so defined. The following result then follows directly from Theorem 2.2 .

Theorem 3.5. Suppose the functional time series $\left\{X_{i}\right\}$ satisfies the assumptions of Theorem 2.2. and the series $\left\{X_{j}^{*}\right\}$ satisfies the same assumptions stated in terms of the $\varepsilon_{j}^{*}$. If (3.2) holds, then (3.7) holds.

We emphasize that under the conditions of Theorem 3.5 relation (3.7) holds both under $H_{0}$ and $H_{A}$.

We now focus on the numerical issues related to the computation of the $\hat{a}_{i}$ and the $\hat{\lambda}_{i}$ appearing in the definitions of statistics $U_{N, M}^{(1)}$ and $U_{N, M}^{(2)}$. The $\hat{a}_{i}$ and the $\hat{\lambda}_{i}$ require the 
computation of the eigenfunctions and the eigenvalues of the operator $\hat{D}$. Except in the case of independent observations in each of the two samples, these quantities cannot be computed using existing software because $\hat{D}$ is not an empirical covariance operator of a functional iid sample. We recommend the following algorithm which we used to implement the tests. Let $\left\{e_{\ell}, \ell \geq 1\right\}$ be an orthonormal basis. The results reported in Section 4 are based on an implementation which uses the Fourier basis. In order to find approximate solutions to

$$
\int \hat{d}_{N, M}(t, s) \phi(s) d s=\lambda \phi(t)
$$

we use approximate expansions for $\phi(s)$ and $\hat{d}_{N, M}(t, s)$ :

$$
\begin{aligned}
\phi(s) & \approx \sum_{k=1}^{49} \phi_{k} e_{k}(t) \\
\hat{d}_{N, M}(t, s) & \approx \sum_{k=1}^{49} \sum_{\ell=1}^{49} d_{k, \ell} e_{k}(t) e_{\ell}(s) .
\end{aligned}
$$

The coefficients $\phi_{k}$ and $d_{k, \ell}$ are given by

$$
\phi_{k}=\int \phi(t) e_{k}(t) d t
$$

and

$$
d_{k, \ell}=\iint \hat{d}_{N, M}(t, s) e_{k}(t) e_{\ell}(s) d t d s
$$

We replace $\phi(s)$ and $\hat{d}_{N, M}(t, s)$ in the left side of 3.17 with the above expansions to obtain

$$
\sum_{k=1}^{49} \sum_{\ell=1}^{49} d_{k, \ell} e_{k}(t) \approx \lambda \phi(t) .
$$

Multiplying both sides of 3.19 by $e_{j}$ and integrating yields

$$
\sum_{\ell=1}^{49} d_{j, \ell} \phi_{\ell} \approx \lambda \phi_{j}, \quad 1 \leq j \leq 49 .
$$

In matrix form this is

$$
\mathrm{D} \phi=\lambda \phi
$$

where $\mathbf{D}=\left[d_{j, \ell}, \quad 1 \leq j, \ell \leq 49\right]$ and $\phi=\left[\phi_{1}, \phi_{2}, \ldots, \phi_{49}\right]^{T}$. Thus we have reduced the problem of finding solutions of (3.17) to finding eigenvalues and eigenvectors of the 
matrix D. Let $\left[\phi_{m, 1}, \phi_{m, 2}, \ldots, \phi_{m, 49}\right]^{T}$ be the eigenvector corresponding to the $m$ th largest eigenvalue of $\mathbf{D}$. Then the eigenfunction associated with the $m$ th largest eigenvalue of $\hat{d}_{N, M}(t, s)$ is approximately $\sum_{\ell}^{49} \phi_{m, \ell} e_{\ell}(t)$. Using this notation, we obtain

$$
\begin{aligned}
\hat{a}_{i} & =\left\langle\bar{X}_{N}-\bar{X}_{M}^{*}, \hat{\varphi}_{i}\right\rangle \\
& \approx \sum_{\ell=1}^{49} \phi_{m, \ell}\left(N^{-1} \sum_{i=1}^{N}\left\langle X_{i}, e_{\ell}\right\rangle-M^{-1} \sum_{j=1}^{M}\left\langle X_{j}^{*}, e_{\ell}\right\rangle\right) .
\end{aligned}
$$

We then obtain $U_{N, M}^{(1)}$ and $U_{N, M}^{(2)}$ as per 3.11) and (3.12).

To complete the description of the test procedures, we must specify how the value of $p$ in the definitions of $U_{N, M}^{(1)}$ and $U_{N, M}^{(2)}$ is selected. This issue has been extensively studied in one sample problems, and several approaches have been put forward, including cross validation and penalty criteria. In our experience (for smooth densely recorded curves), the simple cumulative variance method advocated by Ramsay and Silverman (2005) has been satisfactory. We therefore recommend to use $p$ such that the first $p$ empirical functional principal components in each sample explain about $85 \%$ of the variance. As we will see in the data examples studied in Section 4, it is typically useful to look at the $\mathrm{P}$-values for a range of $p$ 's.

\section{A simulation study and data examples}

We begin by presenting the results of a simulation study intended to evaluate the empirical size and power of the testing procedures introduced in Section 3 . We then illustrate their properties on two data examples.

\subsection{A simulation study}

In this section, we compare the the performance of the tests based on statistics $U_{N, M}^{(1)}$ and $U_{N, M}^{(2)}$ using simulated Gaussian functional data. We consider all combinations of sample sizes $N, M=50,100,100$, and each pair of data generated processes was replicated three thousand times. To investigate the empirical size, without loss of generality, we set $\mu(t)=\mu^{*}(t)=0$. Under the alternative, we set $\mu(t)=0$ and $\mu^{*}(t)=a t(1-t)$. The power is then a function of the parameter $a$. We considered two settings for the errors:

1. Both the $\varepsilon_{i}(t)$ and the $\varepsilon_{j}^{*}(t)$ are iid Brownian bridges.

2. Both the $\varepsilon_{i}(t)$ and the $\varepsilon_{j}^{*}(t)$ are functional $\operatorname{AR}(1)(\operatorname{FAR}(1))$ processes with the kernel,

$$
\psi(t, s)=\frac{e^{-\left(t^{2}+s^{2}\right) / 2}}{4 \int_{0}^{1} e^{-x^{2}} d x} .
$$


That is, the error terms, $\varepsilon_{i}(t)$, follow the model

$$
\varepsilon_{i}(t)=\int_{0}^{1} \psi(t, s) \varepsilon_{i-1}(s) d s+B_{i}(t)
$$

where $B_{i}(t)$ are iid Brownian bridges.

We calculated the test statistics $U_{N, M}^{(1)}$ and $U_{N, M}^{(2)}$ as explained in Section 3. These statistics depend on the choice of the weight functions $K$ and $K^{*}$, and the bandwidth functions $h$ and $h^{*}$. A great deal of attention has been devoted over several decades to the optimal selection of these functions for scalar and vector-valued time series, and we cannot address this issue within the space of this paper. We follow the recommendation of ? and use, for both samples, the flat top kernel

$$
K(t)= \begin{cases}1 & 0 \leq|t|<0.1 \\ 1.1-|t| & 0.1 \leq|t|<1.1 \\ 0 & 1.1 \leq|t|\end{cases}
$$

with $h=N^{1 / 3}$ and $h^{*}=M^{1 / 3}$. We emphasize that this full estimation procedure was used for all data generating processes, including those with independent errors.

TABLE 4.1. Power of Test (in \%) using $U_{100,200}^{(1)}$ and $U_{100,200}^{(2)}$ with iid Brownian-bridge errors

\begin{tabular}{|c|r|r|r|r|r|r|}
\hline & \multicolumn{2}{|c|}{$\alpha=.01$} & \multicolumn{2}{c|}{$\alpha=.05$} & \multicolumn{2}{c|}{$\alpha=.10$} \\
\hline$a$ & $U_{100,200}^{(1)}$ & $U_{100,200}^{(2)}$ & $U_{100,200}^{(1)}$ & $U_{100,200}^{(2)}$ & $U_{100,200}^{(1)}$ & $U_{100,200}^{(2)}$ \\
\hline 0.0 & 1.5 & 1.5 & 6.3 & 6.2 & 11.4 & 11.6 \\
\hline 0.1 & 2.5 & 3 & 7.4 & 8.0 & 13.2 & 13.6 \\
\hline 0.2 & 6.0 & 4.4 & 16.7 & 13.0 & 24.8 & 20.2 \\
\hline 0.3 & 14.2 & 9.2 & 30.4 & 23.2 & 41.3 & 33.0 \\
\hline 0.4 & 26.4 & 17.0 & 48.5 & 36.1 & 60.8 & 48.0 \\
\hline 0.5 & 44.0 & 31.4 & 64.7 & 53.5 & 75.2 & 64.3 \\
\hline 0.6 & 59.4 & 45.9 & 80.3 & 68.0 & 87.9 & 78.4 \\
\hline 0.7 & 78.0 & 64.7 & 91.8 & 82.4 & 96.0 & 89.0 \\
\hline 0.8 & 88.0 & 78.0 & 95.9 & 90.9 & 98.0 & 94.6 \\
\hline 0.9 & 94.2 & 88.1 & 98.7 & 95.8 & 99.4 & 97.9 \\
\hline 1.0 & 98.0 & 94.8 & 99.5 & 98.5 & 99.9 & 99.3 \\
\hline 1.1 & 99.6 & 98.4 & 100.0 & 99.8 & 100.0 & 100.0 \\
\hline 1.2 & 99.9 & 99.4 & 100.0 & 99.9 & 100.0 & 100.0 \\
\hline 1.3 & 100.0 & 99.8 & 100.0 & 100.0 & 100.0 & 100.0 \\
\hline
\end{tabular}


TABLE 4.2. Power of Test (in \%) using $U_{100,200}^{(1)}$ and $U_{100,200}^{(2)}$ with $\operatorname{FAR}(1)$ errors

\begin{tabular}{|c|r|r|r|r|r|r|}
\hline & \multicolumn{2}{|c|}{$\alpha=.01$} & \multicolumn{2}{c|}{$\alpha=.05$} & \multicolumn{2}{c|}{$\alpha=.10$} \\
\hline$a$ & $U_{100,200}^{(1)}$ & $U_{100,200}^{(2)}$ & $U_{100,200}^{(1)}$ & $U_{100,200}^{(2)}$ & $U_{100,200}^{(1)}$ & $U_{100,200}^{(2)}$ \\
\hline 0.0 & 1.8 & 1.9 & 6.6 & 7.2 & 12.2 & 13.5 \\
\hline 0.1 & 2.4 & 2.2 & 7.9 & 7.7 & 13.5 & 14.5 \\
\hline 0.2 & 5.1 & 3.3 & 13.6 & 11.6 & 21.6 & 18.7 \\
\hline 0.3 & 9.8 & 6.3 & 23.6 & 17.6 & 34.5 & 26.8 \\
\hline 0.4 & 19.4 & 12.3 & 35.9 & 26.5 & 46.7 & 36.3 \\
\hline 0.5 & 26.8 & 19.5 & 47.9 & 38.6 & 60.4 & 49.7 \\
\hline 0.6 & 42.1 & 29.6 & 62.2 & 51.8 & 73.1 & 62.5 \\
\hline 0.7 & 56.4 & 42.8 & 75.4 & 63.8 & 83.2 & 74.0 \\
\hline 0.8 & 68.6 & 53.8 & 85.7 & 74.6 & 91.5 & 83.1 \\
\hline 0.9 & 80.8 & 67.6 & 92.7 & 85.9 & 96.4 & 91.9 \\
\hline 1.0 & 87.4 & 78.7 & 95.9 & 90.8 & 98.1 & 94.5 \\
\hline 1.1 & 93.7 & 86.8 & 97.9 & 95.8 & 99.1 & 97.6 \\
\hline 1.2 & 97.6 & 93.7 & 99.5 & 98.1 & 99.8 & 99.2 \\
\hline 1.3 & 98.5 & 96.4 & 99.7 & 98.9 & 99.9 & 99.6 \\
\hline
\end{tabular}

The results of the simulation study can be summarized as follows. The empirical size of the tests is larger in the case of $\operatorname{FAR}(1)$ errors. When $a$ increases to 0.2 or larger, the empirical power of the test is smaller in the case of $\operatorname{FAR}(1)$ errors. Thus increasing the dependence in the error terms increases the size and decreases the power of the test. In both cases the tests have have a slightly larger-than-nominal size and very good power. These observations are illustrated in Tables 4.1 and 4.2. Based on the whole simulation study, we can conclude that the performance of both tests is better if the sample sizes $N$ and $M$ are about equal. For example, for $N=M=100$, the empirical sizes are closer to the nominal sizes then in the case $N=100, M=200$ shown in Tables 4.1 and 4.2 . The power is very high even for small sample sizes. This is illustrated in Figure 4.1 which shows the samples with $N=M=50$ and with slightly different means $(a=0.8)$. Visual inspection does not readily lead to the conclusion that the samples in the left and right panels of Figure 4.1 have different means, yet our tests can detect it with a very high probability. None of the two test statistics clearly dominates the other for the simulated Gaussian data, but a difference in behavior can be see when the tests are applied to real data sets, to which we now turn. 
FIGURE 4.1. Fifty trajectories of the Brownian bridge (left) and fifty independent trajectories of the Brownian bridge plus $\mu^{*}(t)=0.8 t(1-t)$ (right). The tests can detect the different means with probability close to $90 \%$.
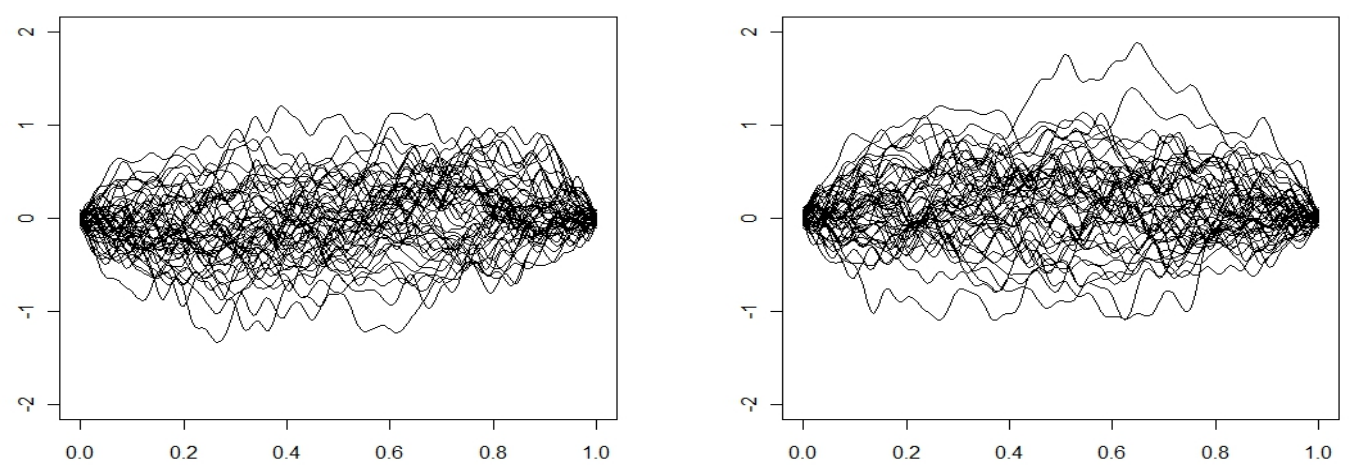

\subsection{Mediterranean fruit flies}

In our first example, it can be assumed that the curves in each sample are independent, as they were obtained from a randomized experiment. The data set used in this example was kindly made available to us by Hans-Georg Müller. It was extensively studied in biological and statistical literature, see Müller and Stadtmüller (2005) and references therein. We consider 534 egg-laying curves (count of eggs per unit time interval) of medflies who lived at least 30 days. Each function is defined over an interval [0,30], and its value on day $t \leq 30$ is the count of eggs laid by fly $i$ on that day. The 534 flies are classified into long-lived, i.e. those who lived longer than 44 days, and short-lived, i.e. those who died before the end of the 44th day after birth. In the data set, there are 256 short-lived, and 278 long-lived flies. This classification naturally defines two samples: Sample 1: the egg-laying curves $\left\{X_{i}(t), 0<t \leq 30, i=1,2, \ldots, 256\right\}$ of the short-lived flies. Sample 2: the egg-laying curves $\left\{X_{j}^{*}(t), 0<t \leq 30, j=1,2, \ldots, 278\right\}$ of the long-lived flies. The egg-laying curves are very irregular; Figure 4.2 shows ten smoothed curves of short- and long-lived flies. The tests are applied to such smooth trajectories.

Table 4.3 shows the $\mathrm{P}$-values as a function of $p$. For both samples, $p=2$ explains slightly over $85 \%$ of the variance, so this is the value we would recommend using. Both tests reject the equality of the mean functions, even though the sample means, shown in Figure 4.3 , are not far apart. The P-values for the statistic $U^{(1)}$ are much more stable, equal to about $1 \%$, no matter the value of $p$. The behavior of the test based on $U^{(2)}$ is more erratic. This indicates that while the test based on $U^{(2)}$ is easier to apply because it uses standard chi-square critical values, the test based on $U^{(1)}$ may be more reliable. 
FIGURE 4.2. Ten randomly selected smoothed egg-laying curves of short-lived medflies (left panel), and ten such curves for long-lived medfies (right panel).
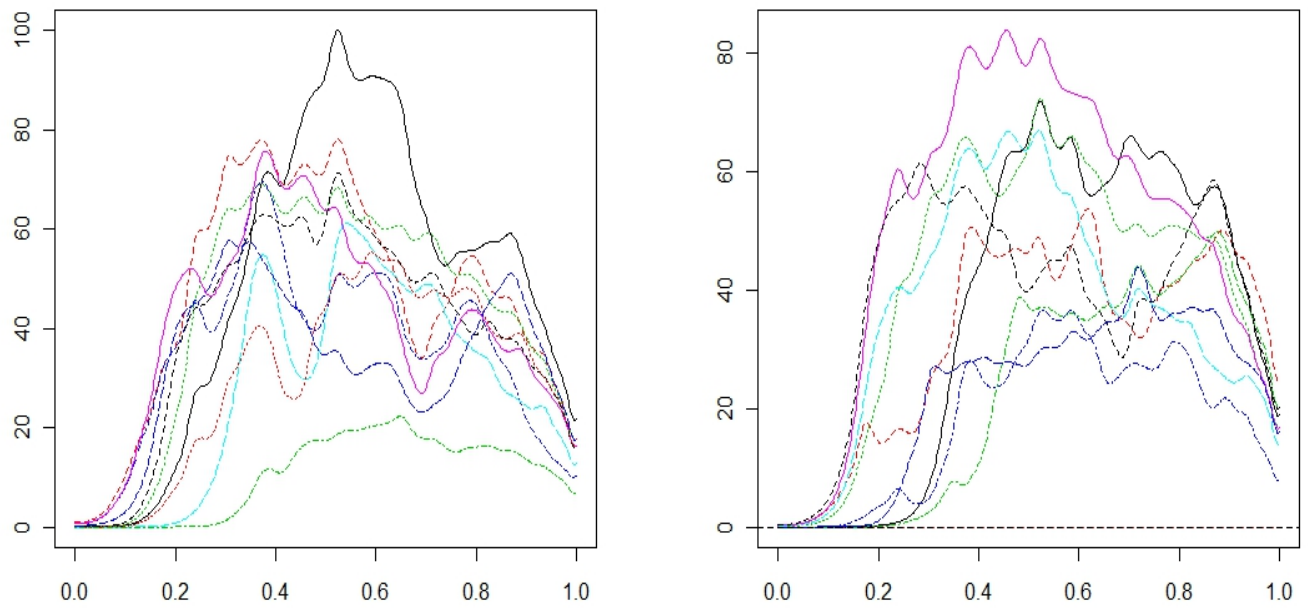

TABLE 4.3. P-values (in percent) of the tests based on statistics $U_{N, M}^{(2)}$ and $U_{N, M}^{(1)}$ applied to medfly data.

\begin{tabular}{|c|rrrrrrrrr|}
\hline$p$ & 1 & 2 & 3 & 4 & 5 & 6 & 7 & 8 & 9 \\
\hline$U^{(1)}$ & 1.0 & 1.0 & 1.0 & 1.1 & 1.1 & 1.0 & 1.0 & 1.1 & 1.1 \\
$U^{(2)}$ & 1.0 & 2.2 & 3.0 & 5.7 & 10.3 & 15.3 & 3.2 & 2.7 & 5.0 \\
\hline
\end{tabular}


FiguRE 4.3. Estimated mean functions for the medfly data: short lived -solid line; long lived -dashed line.

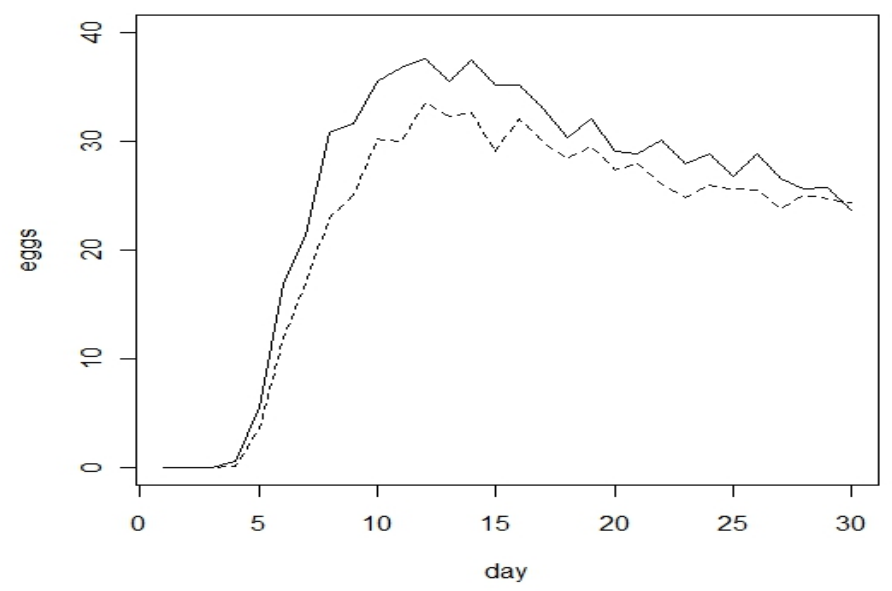

\subsection{Eurodollar futures contracts}

Our next example uses financial data kindly made available by Vladislav Kargin. This data is used as an example of modeling with the functional AR(1) process in Kargin and Onatski (2008), The curves, one curve per day, are constructed from the prices of Eurodollar futures contracts with decreasing expiration dates. The seller of a Eurodollar futures contract takes on an obligation to deliver a deposit of one million US dollars to a bank account outside the United States $i$ months from today. The price the buyer is willing to pay for this contract depends on the prevailing interest rate. These contracts are traded on the Chicago Mercantile Exchange and provide a way to lock in an interest rate. Eurodollar futures are a liquid asset and are responsive to the Federal Reserve policy, inflation, and economic indicators.

The data we study consist of 114 points per day; point $i$ corresponds to the price of a contract with closing date $i$ months from today. We consider 4 samples, each consisting of 100 days of this data:

Sample 1: curves from September 7, 1999 to January 27, 2000.

Sample 2: curves from January 24, 1997 to June 17, 1997.

Sample 3: curves from December 4, 1995 to April 24, 1996.

Sample 4: curves from March 6, 2001 to July 26, 2001.

Figure 4.4 shows the sample mean functions for the four samples. If a significance test does not reject $H_{0}$, we can conclude that the expectations of the future evolution of interest 
rates are the same for the two periods over which the samples were taken. A rejection means that these expectations are significantly different. As the analysis below reveals, we can conclude that expectations of future interest rates were different in Spring 1996 than in Summer 2001.

FigurE 4.4. Sample means of the Eurodollar curves: Left: sample 1 solid; sample 2 dashed. Right: sample 3 solid; sample 4 dashed.
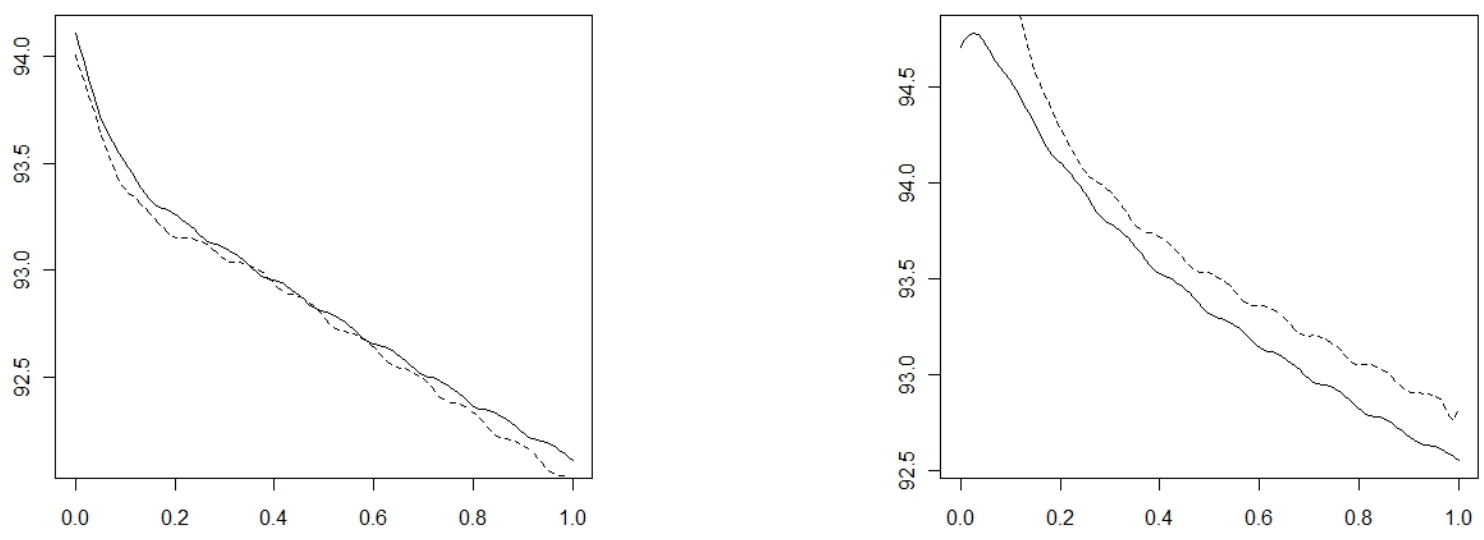

Table 4.4 shows the $\mathrm{P}$-values as a function of $p$ when the test is applied to samples 1 and 2 , and also when it is applied to samples 3 and 4 . In both samples 1 and $2, p=1$ explains more than $94 \%$ of the variance, in both samples 3 and $4, p=1$ explains more than $84 \%$ of the variance. Thus, following the recommendation of Section 3 , we use the $\mathrm{P}$-values obtained with $p=1$. They lead to the acceptance of the null hypothesis of the equality of mean functions for periods corresponding to samples 1 and 2, and to its rejection for periods corresponding to samples 3 and 4 (notice that the 0.81 in the bottom panel of Table 4.4 is $0.81 \%$ ). These conclusions agree with a visual evaluation of the sample mean functions in Figure 4.4. They also confirm the observation made in Section 4.1 that the tests have very good power, as the curves in the right panel of Figure 4.4 are not far apart. Both graphs in Figure 4.4 give us an idea what kind of differences in the sample mean functions are statistically significant, and which are not.

\subsection{Conclusions}

The simulations and data examples of this section show the tests we propose enjoy good finite sample properties. Tests of this type allow us to quantify statistical significance of conjectures made on the basis of exploratory analysis. For example, the sample mean curves in Figure 4.3 and the right panel of Figure 4.4 look a bit different, but a significance 
TABLE 4.4. P-values (in percent) of the statistics applied to Eurodollar data; samples 1 and 2 (top), samples 3 and 4 (bottom).

\begin{tabular}{|c|rrrrr|}
\hline$p$ & 1 & 2 & 3 & 4 & 5 \\
\hline$U^{(1)}$ & 38.49 & 39.17 & 37.12 & 37.15 & 35.50 \\
$U^{(2)}$ & 38.49 & 68.52 & 0.00 & 0.00 & 0.00 \\
\hline
\end{tabular}

\begin{tabular}{|c|rrrrr|}
\hline$p$ & 1 & 2 & 3 & 4 & 5 \\
\hline$U^{(1)}$ & 0.81 & 0.23 & 0.10 & 0.01 & 0.07 \\
$U^{(2)}$ & 0.81 & 0.00 & 0.00 & 0.00 & 0.00 \\
\hline
\end{tabular}

test allows us to state with confidence that they correspond to different population mean functions.

In many procedures of functional data analysis, both exploratory and inferential, the issue of choosing an optimal dimension reduction parameter, like the $p$ in our setting, is delicate. Therefore, procedures less sensitive to such a choice are preferable. From this angle, the Monte Carlo test based $U^{(1)}$ is preferable, as an inspection of Tables 4.3 and 4.4 reveals. The test based on $U^{(2)}$ is however easier to apply, and in our examples and simulations leads to the same conclusions if $p$ is chosen according to the cumulative variance rule.

The data examples of this section also show that the optimal value of $p$ is typically a small single digit number, 1 or 2 in our examples. Therefore, developing asymptotics as $p$ tends to infinity is not necessary, and may, in fact, be misleading because for larger values of $p$ the tests may yield counterintuitive results.

\section{Proofs of the results of Section 2}

Proof of TheOrem 2.1. The proof is done in two steps. First we show that $N^{-1 / 2} \sum_{i=1}^{N} \varepsilon_{i}(t)$ is close to $N^{-1 / 2} \sum_{i=1}^{N} \varepsilon_{i, m}(t)$, if $m$ is sufficiently large. Then we establish 2.3 for $m$ dependent functions for any $m \geq 1$.

As the first step, we show that

$$
\limsup _{m \rightarrow \infty} \limsup _{N \rightarrow \infty} E \int\left[N^{-1 / 2} \sum_{i=1}^{N}\left(\varepsilon_{i}(t)-\varepsilon_{i, m}(t)\right)\right]^{2} d t=0,
$$


where the variables $\varepsilon_{i, m}$ are defined in $(1.3)$. By stationarity,

$$
\begin{aligned}
E & {\left[\sum_{1 \leq i \leq N}\left(\varepsilon_{i}(t)-\varepsilon_{i, m}(t)\right)\right]^{2} } \\
& =\sum_{1 \leq i \leq N} \sum_{1 \leq j \leq N} E\left(\varepsilon_{i}(t)-\varepsilon_{i, m}(t)\right)\left(\varepsilon_{j}(t)-\varepsilon_{j, m}(t)\right) \\
& =N E\left(\varepsilon_{0}(t)-\varepsilon_{0, m}(t)\right)^{2}+2 \sum_{1 \leq i<j \leq N} E\left(\varepsilon_{i}(t)-\varepsilon_{i, m}(t)\right)\left(\varepsilon_{j}(t)-\varepsilon_{j, m}(t)\right) .
\end{aligned}
$$

In the proof, we will repeatedly use independence relations which follow from representations (1.1) and (1.3). First observe that if $j>i$, then $\left(\varepsilon_{i}, \varepsilon_{i, m}\right)$ is independent of $\varepsilon_{j, j-i}$ because

$$
\varepsilon_{j, j-i}=f\left(\delta_{j}, \ldots, \delta_{i+1}, \delta_{j, i}^{(j-i)}, \delta_{j, i-1}^{(j-i)}, \ldots\right) .
$$

Consequently, $E\left(\varepsilon_{i}(t)-\varepsilon_{i, m}(t)\right) \varepsilon_{j, j-i}(t)=0$, and so

$$
\sum_{1 \leq i<j \leq N} E\left(\varepsilon_{i}(t)-\varepsilon_{i, m}(t)\right) \varepsilon_{j}(t)=\sum_{1 \leq i<j \leq N} E\left(\varepsilon_{i}(t)-\varepsilon_{i, m}(t)\right)\left(\varepsilon_{j}(t)-\varepsilon_{j, j-i}(t)\right) .
$$

Using the Cauchy-Schwarz inequality and 1.2 , we conclude

$$
\begin{array}{rl}
\mid \int \sum_{1 \leq i<j \leq N} E & E\left(\varepsilon_{i}(t)-\varepsilon_{i, m}(t)\right)\left(\varepsilon_{j}(t)-\varepsilon_{j, j-i}(t)\right) d t \mid \\
& \leq \sum_{1 \leq i<j \leq N} \int\left[E\left(\varepsilon_{i}(t)-\varepsilon_{i, m}(t)\right)^{2}\right]^{1 / 2}\left[E\left(\varepsilon_{j}(t)-\varepsilon_{j, j-i}(t)\right)^{2}\right]^{1 / 2} d t \\
& \leq \sum_{1 \leq i<j \leq N}\left[\int E\left(\varepsilon_{i}(t)-\varepsilon_{i, m}(t)\right)^{2} d t\right]^{1 / 2}\left[\int E\left(\varepsilon_{j}(t)-\varepsilon_{j, j-i}(t)\right)^{2} d t\right]^{1 / 2} \\
& =\sum_{1 \leq i<j \leq N}\left[\int E\left(\varepsilon_{0}(t)-\varepsilon_{0, m}(t)\right)^{2} d t\right]^{1 / 2}\left[\int E\left(\varepsilon_{0}(t)-\varepsilon_{0, j-i}(t)\right)^{2} d t\right]^{1 / 2} \\
& \leq N\left[\int E\left(\varepsilon_{0, m}(t)-\varepsilon_{0}(t)\right)^{2} d t\right]^{1 / 2} \sum_{k \geq 1}\left[\int\left(\varepsilon_{0}(t)-\varepsilon_{0, k}(t)\right)^{2} d t\right]^{1 / 2} .
\end{array}
$$

Hence

$$
\limsup _{m \rightarrow \infty} \limsup _{N \rightarrow \infty} \frac{1}{N}\left|\int \sum_{1 \leq i<j \leq N} E\left[\left(\varepsilon_{i, m}(t)-\varepsilon_{i}(t)\right) \varepsilon_{j}(t)\right] d t\right|=0 .
$$

Similar arguments give

$$
\limsup _{m \rightarrow \infty} \limsup _{N \rightarrow \infty} \frac{1}{N}\left|\int \sum_{1 \leq i<j \leq N} E\left[\left(\varepsilon_{i, m}(t)-\varepsilon_{i}(t)\right) \varepsilon_{j, m}(t)\right] d t\right|=0 .
$$


Completing the verification of (5.1).

The next the step is to show that $N^{-1 / 2} \sum_{1 \leq i \leq N} \varepsilon_{i, m}$ converges to a Gaussian process $Z_{m}$ with covariances defined analogously to $(2.4)$. Recall that for every integer $m \geq 1,\left\{\varepsilon_{i, m}\right\}$ is an $m$-dependent sequence of functions. To lighten the notation, in the remainder of the proof, we fix $m$ and denote sequence $\left\{\varepsilon_{i, m}\right\}$ by $\left\{\varepsilon_{i}\right\}$, so $\left\{\varepsilon_{i}\right\}$ is now $m$-dependent.

Let $K>1$ be an integer and $\psi_{i}$ be an orthonormal basis determined by the eigenfunctions of $E \varepsilon(t) \varepsilon(s)$. The corresponding eigenvalues are denoted by $\nu_{i}$. Then, by the KarhunenLoéve expansion, we have

$$
\varepsilon_{i}(t)=\sum_{\ell \geq 1}\left\langle\varepsilon_{i}, \psi_{\ell}\right\rangle \psi_{\ell}(t) .
$$

Next we define

$$
\varepsilon_{i}^{(K)}(t)=\sum_{1 \leq \ell \leq K}\left\langle\varepsilon_{i}, \psi_{\ell}\right\rangle \psi_{\ell}(t) .
$$

By the triangle inequality we have that

$$
\begin{aligned}
& \left\{E \int\left[\sum_{1 \leq i \leq N}\left(\left(\varepsilon_{i}(t)-\varepsilon_{i}^{(K)}(t)\right)\right]^{2} d t\right\}^{1 / 2}\right. \\
& \leq\left\{E \int\left[\sum_{i \in V(0)}\left(\left(\varepsilon_{i}(t)-\varepsilon_{i}^{(K)}(t)\right)\right]^{2} d t\right\}^{1 / 2}+\ldots\right. \\
& +\left\{E \int\left[\sum_{i \in V(m-1)}\left(\left(\varepsilon_{i}(t)-\varepsilon_{i}^{(K)}(t)\right)\right]^{2} d t\right\}^{1 / 2},\right.
\end{aligned}
$$

where $V(k)=\{i: 1 \leq i \leq N, i=k(\bmod m)\}, 0 \leq k \leq m-1$. Due to the $m$ dependence of the sequence $\left\{\varepsilon_{i}\right\}, \sum_{i \in V(k)}\left(\varepsilon_{i}(t)-\varepsilon_{i}^{(K)}(t)\right)$ is a sum of independent, identically distributed random variables, and thus we get that

$$
E \int\left[\sum_{i \in V(m-1)}\left(\left(\varepsilon_{i}(t)-\varepsilon_{i}^{(K)}(t)\right)\right]^{2} d t \leq N \sum_{\ell \geq K} E\left\langle X_{0}, \psi_{\ell}\right\rangle^{2} .\right.
$$

Utilizing

$$
\lim _{K \rightarrow \infty} \sum_{\ell \geq K} E\left\langle X_{0}, \psi_{\ell}\right\rangle^{2}=0
$$

we conclude that for any $x>0$

$$
\limsup _{K \rightarrow \infty} \limsup _{N \rightarrow \infty} P\left\{\int\left[\frac{1}{N^{1 / 2}} \sum_{1 \leq i \leq N}\left(\left(\varepsilon_{i}(t)-\varepsilon_{i}^{(K)}(t)\right)\right]^{2} d t>x\right\}=0 .\right.
$$


The sum of the $\varepsilon_{i}^{(K)}$ 's can be written as

$$
\frac{1}{N^{1 / 2}} \sum_{1 \leq i \leq N} \varepsilon_{i}^{(K)}(t)=\sum_{1 \leq \ell \leq K} \psi_{\ell}(t) \frac{1}{N^{1 / 2}} \sum_{1 \leq i \leq N}\left\langle\varepsilon_{i}, \psi_{\ell}\right\rangle .
$$

Next, we use the central limit theorem for stationary $m$-dependent sequences of random vectors (see ? and the Cramér-Wold theorems in ?, pages 9 and 120)) and and get that

$$
\left\{\frac{1}{N^{1 / 2}} \sum_{1 \leq i \leq N}\left\langle\varepsilon_{i}, \psi_{\ell}\right\rangle, 1 \leq \ell \leq K\right\}^{T} \stackrel{d}{\rightarrow} \mathbf{N}_{K}\left(\mathbf{0}, \boldsymbol{\Delta}_{K}\right)
$$

where $\mathbf{N}_{K}\left(\mathbf{0}, \boldsymbol{\Delta}_{K}\right)$ is a $K$-dimensional normal random variable with zero mean and covariance matrix $\boldsymbol{\Delta}_{K}=\operatorname{diag}\left(\nu_{1}, \ldots, \nu_{K}\right)$. Thus we proved that for all $K>1$

$$
N^{-1 / 2} \sum_{1 \leq i \leq N} \varepsilon_{i}^{(K)}(t) \stackrel{d}{\rightarrow} \sum_{1 \leq \ell \leq K} \nu_{\ell}^{1 / 2} N_{\ell} \psi_{\ell}(t) \quad \text { in } L^{2}
$$

where $N_{i}, i \geq 1$ are independent standard normal random variables. It is easy to see that

$$
\int\left(\sum_{K<\ell<\infty} \nu_{\ell}^{1 / 2} N_{\ell} \psi_{\ell}(t)\right)^{2} d t=\sum_{K<\ell<\infty} \nu_{\ell} N_{\ell}^{2} \stackrel{P}{\rightarrow} 0
$$

as $K \rightarrow \infty$. Thus we have the convergence of $N^{-1 / 2} \sum_{1 \leq i \leq N} \varepsilon_{i}$ for any $m$ and therefore the proof of the Theorem is now complete.

Proof of Theorem 2.2. First we reduce (2.15) to (5.2). Then, we reduce (5.2) to 5.6 .

Since

$$
\hat{\gamma}_{0}(t, s)=\frac{1}{N} \sum_{i=1}^{N}\left(X_{i}(t)-\mu(t)\right)\left(X_{i}(s)-\mu(s)\right)-\left(\bar{X}_{N}(t)-\mu(t)\right)\left(\bar{X}_{N}(s)-\mu(s)\right),
$$

we obtain that

$$
\begin{aligned}
& \iint\left\{\hat{\gamma}_{0}(t, s)-E\left[\varepsilon_{0}(t) \varepsilon_{0}(s)\right]\right\}^{2} d t d s \\
& \leq 4 \iint\left\{\frac{1}{N} \sum_{i=1}^{N}\left(X_{i}(t)-\mu(t)\right)\left(X_{i}(s)-\mu(s)\right)-E\left[\varepsilon_{0}(t) \varepsilon_{0}(s)\right]\right\}^{2} d t d s \\
& \quad+4\left(\int\left(\bar{X}_{N}(t)-\mu(t)\right)^{2} d t\right)^{2} \\
& =o_{P}(1)
\end{aligned}
$$


using the ergodic theorem for random variables in a Hilbert space.

Next observe that

$$
\begin{aligned}
\hat{\gamma}_{i}(t, s)= & \frac{1}{N} \sum_{j=i+1}^{N} \varepsilon_{j}(t) \varepsilon_{j-i}(s) \\
& +\frac{N-i}{N} \bar{\varepsilon}_{N}(t) \bar{\varepsilon}_{N}(s)-\left(\frac{1}{N} \sum_{j=i+1}^{N} \varepsilon_{j}(t)\right) \bar{\varepsilon}_{N}(s)-\bar{\varepsilon}_{N}(t)\left(\frac{1}{N} \sum_{j=i+1}^{N} \varepsilon_{j-i}(s)\right) .
\end{aligned}
$$

Therefore, setting,

$$
\bar{\gamma}_{i}(t, s)=\frac{1}{N} \sum_{j=i+1}^{N} \varepsilon_{j}(t) \varepsilon_{j-i}(s) .
$$

we obtain

$$
\begin{aligned}
\sum_{i=1}^{N-1} K\left(\frac{i}{h}\right) \hat{\gamma}_{i}(t, s)= & \sum_{i=1}^{N-1} K\left(\frac{i}{h}\right) \bar{\gamma}_{i}(t, s) \\
& -\sum_{i=1}^{N-1} K\left(\frac{i}{h}\right)\left\{\frac{1}{N} \sum_{j=i+1}^{N} \varepsilon_{j}(t)\right\} \bar{\varepsilon}_{N}(s) \\
& -\sum_{i=1}^{N-1} K\left(\frac{i}{h}\right)\left\{\frac{1}{N} \sum_{j=i+1}^{N} \varepsilon_{j-i}(s)\right\} \bar{\varepsilon}_{N}(t) \\
& +\sum_{i=1}^{N-1} K\left(\frac{i}{h}\right) \frac{N-i}{N} \bar{\varepsilon}_{N}(t) \bar{\varepsilon}_{N}(s) .
\end{aligned}
$$

gud

By stationarity we conclude that for any $1 \leq i \leq N$,

$$
\begin{aligned}
E \int\left(\frac{1}{N} \sum_{j=i+1}^{N} \varepsilon_{j}(t)\right)^{2} d t & =\frac{1}{N^{2}} \sum_{j=i+1}^{N} \int E \varepsilon_{0}^{2}(t) d t+\frac{2}{N^{2}} \sum_{j=i+1}^{N}(N-j) \int E \varepsilon_{0}(t) \varepsilon_{j}(t) d t \\
& \leq \frac{1}{N} \int E \varepsilon_{0}^{2}(t) d t+\frac{4}{N} \sum_{j=1}^{\infty}\left|\int E \varepsilon_{0}(t) \varepsilon_{j}(t) d t\right| .
\end{aligned}
$$


Since $\varepsilon_{0}$ and $\varepsilon_{j, j}$ are independent, we get by 1.2

$$
\begin{aligned}
\sum_{j=1}^{\infty}\left|\int E \varepsilon_{0}(t) \varepsilon_{j}(t) d t\right| & =\sum_{j=1}^{\infty}\left|\int E \varepsilon_{0}(t)\left(\varepsilon_{j}(t)-\varepsilon_{j, j}(t)\right) d t\right| \\
& \leq \sum_{j=1}^{\infty} E\left\{\left(\int \varepsilon_{0}^{2}(t) d t\right)^{1 / 2}\left(\int\left(\varepsilon_{j}(t)-\varepsilon_{j, j}(t)\right)^{2} d t\right)^{1 / 2}\right\} \\
& \leq\left(E \int \varepsilon_{0}^{2}(t) d t\right)^{1 / 2} \sum_{j=1}^{\infty}\left(\int E\left(\varepsilon_{j}(t)-\varepsilon_{j, j}(t)\right)^{2} d t\right)^{1 / 2} \\
& <\infty .
\end{aligned}
$$

Thus, we have

$$
\max _{1 \leq i \leq N} E \int\left(\frac{1}{N} \sum_{j=i+1}^{N} \varepsilon_{j}(t)\right)^{2} d t=O(1) .
$$

Consequently, using the triangle inequality,

$$
\begin{aligned}
& E\left\{\iint\left(\sum_{i=1}^{N-1} K\left(\frac{i}{h}\right)\left[\frac{1}{N} \sum_{j=i+1}^{N} \varepsilon_{j}(t)\right] \bar{\varepsilon}_{N}(s)\right)^{2} d t d s\right\}^{1 / 2} \\
& \leq \sum_{i=1}^{N-1}\left|K\left(\frac{i}{h}\right)\right| E\left\{\left(\int\left[\frac{1}{N} \sum_{j=i+1}^{N} \varepsilon_{j}(t)\right]^{2} d t\right)^{1 / 2}\left(\int \bar{\varepsilon}_{N}^{2}(s) d s\right)^{1 / 2}\right\} \\
& \leq \sum_{i=1}^{N-1}\left|K\left(\frac{i}{h}\right)\right|\left(E \int\left[\frac{1}{N} \sum_{j=i+1}^{N} \varepsilon_{j}(t)\right]^{2}\right)^{1 / 2}\left(\int \bar{\varepsilon}_{N}^{2}(s) d s\right)^{1 / 2} \\
& =\frac{h}{N} O(1)=o(1),
\end{aligned}
$$

on account of 2.13).

Hence to establish 2.15), it is enough to prove that

$$
\iint\left(\sum_{i=1}^{N-1} K\left(\frac{i}{h}\right) \bar{\gamma}_{i}(t, s)-c_{1}(t, s)\right)^{2} d t d s=o_{P}(1),
$$

where

$$
c_{1}(t, s)=\sum_{i \geq 1} E\left[\varepsilon_{0}(s) \varepsilon_{i}(t)\right] .
$$

Let $\left\{\varepsilon_{n, m},-\infty<n<\infty\right\}$ be the random variables defined in 1.3 , where $m$ is a fixed number. Let

$$
\tilde{\gamma}_{i, m}(t, s)=\frac{1}{N} \sum_{j=i+1}^{N} \varepsilon_{j, m}(t) \varepsilon_{j-i, m}(s) .
$$


We show that for every $m \geq 1$,

$$
\iint\left(\sum_{i=1}^{N-1} K\left(\frac{i}{h}\right) \tilde{\gamma}_{i, m}(t, s)-c_{1}^{(m)}(t, s)\right)^{2} d t d s=o_{P}(1)
$$

where

$$
c_{1}^{(m)}(t, s)=\sum_{i=1}^{\infty} E\left[\varepsilon_{1, m}(s) \varepsilon_{i+1, m}(t)\right] .
$$

We also note that 1.3 and 1.2 imply

$$
\lim _{m \rightarrow \infty} \iint\left(c_{1}^{(m)}(t, s)-c_{1}(t, s)\right)^{2} d t d s=0 .
$$

Since $\left\{\varepsilon_{n, m},-\infty<n<\infty\right\}$ is an $m$-dependent sequence,

$$
c_{1}^{(m)}(t, s)=\sum_{i=1}^{m} E\left[\varepsilon_{1, m}(s) \varepsilon_{i+1, m}(t)\right] .
$$

Using (2.7), 2.8 and (2.13), we get

$$
\max _{1 \leq i \leq m}\left|K\left(\frac{i}{h}\right)-1\right| \rightarrow 0, \quad \text { as } N \rightarrow \infty .
$$

By the ergodic theorem,

$$
\iint\left(\tilde{\gamma}_{i, m}(t, s)-E\left[\varepsilon_{1, m}(s) \varepsilon_{i+1, m}(t)\right]\right)^{2} d t d s=o_{P}(1)
$$

for any fixed $i$. Hence (5.3) is proven, once we have shown that

$$
\iint\left(\sum_{i=m+1}^{N-1} K\left(\frac{i}{h}\right) \tilde{\gamma}_{i, m}(t, s)\right)^{2} d t d s=o_{P}(1)
$$

It is easy to see that

$$
\begin{aligned}
& E \iint\left(\sum_{i=m+1}^{N-1} K\left(\frac{i}{h}\right) \tilde{\gamma}_{i, m}(t, s)\right)^{2} d t d s \\
& =\iint\left(\frac{1}{N^{2}} \sum_{i=m+1}^{h} \sum_{\ell=m+1}^{h} \sum_{k=i+1}^{N-1} \sum_{n=\ell+1}^{N-1} K\left(\frac{i}{h}\right) K\left(\frac{\ell}{h}\right) E\left[\varepsilon_{k, m} \varepsilon_{k-i, m} \varepsilon_{n, m} \varepsilon_{n-\ell, m}\right]\right),
\end{aligned}
$$

provided $h \leq N-1$. The sequence $\left\{\varepsilon_{n, m},-\infty<n<\infty\right\}$ is an $m$-dependent, and therefore $\varepsilon_{k, m}$ and $\varepsilon_{k-i, m}$ are independent, since $i \geq m+1$. Similarly, $\varepsilon_{n, m}$ and $\varepsilon_{n-\ell, m}$ are 
independent. Hence the number of terms when $E\left[\varepsilon_{k, m} \varepsilon_{k-i, m} \varepsilon_{n, m} \varepsilon_{n-\ell, m}\right]$ is different from zero is $O(N h)$. Consequently,

$$
E \iint\left(\sum_{i=m+1}^{N-1} K\left(\frac{i}{h}\right) \tilde{\gamma}_{i, m}(t, s)\right)^{2} d t d s=O\left(\frac{h}{N}\right)=o(1) .
$$

This completes the verification of $(5.5)$.

Next we show that for all $\epsilon>0$,

$$
\lim _{m \rightarrow \infty} \limsup _{N \rightarrow \infty} P\left\{\iint\left(\sum_{i=1}^{N-1} K\left(\frac{i}{h}\right)\left[\bar{\gamma}_{i}(t, s)-\tilde{\gamma}_{i, m}(t, s)\right]\right)^{2} d t d s>\epsilon\right\}=0 .
$$

Using the definitions of the covariances $\bar{\gamma}_{i}(t, s)$ and $\tilde{\gamma}_{i, m}(t, s)$, we consider the decompositions

$$
\begin{aligned}
\frac{1}{N} \sum_{i=1}^{N-1} K\left(\frac{i}{h}\right) \sum_{j=i+1}^{N}\left[\varepsilon_{j}(t) \varepsilon_{j-i}(s)-\varepsilon_{j, m}(t) \varepsilon_{j-i, m}(s)\right] \\
=\frac{1}{N}\left\{\sum_{i=1}^{m}+\sum_{i=m+1}^{h}\right\} K\left(\frac{i}{h}\right) \sum_{j=i+1}^{N}\left[\varepsilon_{j}(t) \varepsilon_{j-i}(s)-\varepsilon_{j, m}(t) \varepsilon_{j-i, m}(s)\right]
\end{aligned}
$$

and

$$
\varepsilon_{j}(t) \varepsilon_{j-i}(s)-\varepsilon_{j, m}(t) \varepsilon_{j-i, m}(s)=\left(\varepsilon_{j}(t)-\varepsilon_{j, m}(t)\right) \varepsilon_{j-i}(s)+\left(\varepsilon_{j-i}(s)-\varepsilon_{j-i, m}(s)\right) \varepsilon_{j, m}(t) .
$$

Clearly,

$$
\begin{aligned}
& \left\{\iint\left(\frac{1}{N} \sum_{i=1}^{m} K\left(\frac{i}{h}\right) \sum_{j=i+1}^{N}\left(\varepsilon_{j}(t)-\varepsilon_{j, m}(t)\right) \varepsilon_{j-i}(s)\right)^{2} d t d s\right\}^{1 / 2} \\
& \leq \frac{1}{N} \sum_{i=1}^{m}\left|K\left(\frac{i}{h}\right)\right|\left\{\int\left(\varepsilon_{j}(t)-\varepsilon_{j, m}(t)\right)^{2} d t\right\}^{1 / 2}\left\{\int \varepsilon_{j-i}^{2}(s) d s\right\}^{1 / 2},
\end{aligned}
$$

so, by 2.14,

$$
\begin{aligned}
E & \left\{\iint\left(\frac{1}{N} \sum_{i=1}^{m} K\left(\frac{i}{h}\right) \sum_{j=i+1}^{N}\left(\varepsilon_{j}(t)-\varepsilon_{j, m}(t)\right) \varepsilon_{j-i}(s)\right)^{2} d t d s\right\}^{1 / 2} \\
& \leq m\left\{E \int\left(\varepsilon_{0}(t)-\varepsilon_{0, m}(t)\right)^{2} d t E \int \varepsilon_{0}^{2}(s) d s\right\}^{1 / 2} \\
& \leq A m\left\{E \int\left(\varepsilon_{0}(t)-\varepsilon_{0, m}(t)\right)^{2} d t\right\}^{1 / 2} \rightarrow 0, \text { as } m \rightarrow \infty,
\end{aligned}
$$


according to (2.14), where $A$ is a constant.

Next we use the decomposition

$$
\varepsilon_{j}(t) \varepsilon_{j-i}(s)=\varepsilon_{j, i}(t) \varepsilon_{j-i}(s)+\left(\varepsilon_{j}(t)-\varepsilon_{j, i}(t)\right) \varepsilon_{j-i}(s)
$$

to get

$$
\begin{gathered}
\left\{\iint\left(\frac{1}{N} \sum_{i=m+1}^{h} K\left(\frac{i}{h}\right) \sum_{j=i+1}^{N}\left(\varepsilon_{j}(t)-\varepsilon_{j, i}(t)\right) \varepsilon_{j-i}(s)\right)^{2} d t d s\right\}^{1 / 2} \\
\leq \frac{1}{N} \sum_{i=m+1}^{\infty} \sum_{j=i+1}^{N}\left\{\int\left(\varepsilon_{j}(t)-\varepsilon_{j, i}(t)\right)^{2} d t\right\}^{1 / 2}\left\{\int \varepsilon_{j-i}^{2}(s) d s\right\}^{1 / 2}
\end{gathered}
$$

Therefore, by 2.2 and 1.2 , we have

$$
\begin{aligned}
& E\left\{\iint\left(\frac{1}{N} \sum_{i=m+1}^{h} K\left(\frac{i}{h}\right) \sum_{j=i+1}^{N}\left(\varepsilon_{j}(t)-\varepsilon_{j, i}(t)\right) \varepsilon_{j-i}(s)\right)^{2} d t d s\right\}^{1 / 2} \\
& \leq \frac{1}{N} \sum_{i=m+1}^{\infty} \sum_{j=i+1}^{N} E\left[\left\{\int\left(\varepsilon_{j}(t)-\varepsilon_{j, i}(t)\right)^{2} d t\right\}^{1 / 2}\left\{\int \varepsilon_{j-i}^{2}(s) d s\right\}^{1 / 2}\right] \\
& \leq \frac{1}{N} \sum_{i=m+1}^{\infty} \sum_{j=i+1}^{N}\left[\int\left(\varepsilon_{j}(t)-\varepsilon_{j, i}(t)\right)^{2} d t\right]^{1 / 2}\left[\int \varepsilon_{j-i}^{2}(s) d s\right]^{1 / 2}\left[\varepsilon^{1 / 2} \rightarrow 0, \quad \text { as } m \rightarrow \infty\right. \\
& \leq A \sum_{i=m+1}^{\infty}\left[\int\left(\varepsilon_{0}(t)-\varepsilon_{0, i}(t)\right)^{2} d t\right]^{1 / 2} \rightarrow 0
\end{aligned}
$$

We have shown so far that for any $\epsilon>0$,

$$
\lim _{m \rightarrow \infty} \limsup _{N \rightarrow \infty} P\left\{\iint\left(\frac{1}{N} \sum_{i=m+1}^{h} K\left(\frac{i}{h}\right) \sum_{j=i+1}^{N} \varepsilon_{j}(t) \varepsilon_{j-i}(s)\right)^{2} d t d s>\epsilon\right\}=0 .
$$

Similar arguments give

$$
\lim _{m \rightarrow \infty} \limsup _{N \rightarrow \infty} P\left\{\iint\left(\frac{1}{N} \sum_{i=m+1}^{h} K\left(\frac{i}{h}\right) \sum_{j=i+1}^{N} \varepsilon_{j, m}(t) \varepsilon_{j-i, m}(s)\right)^{2} d t d s>\epsilon\right\}=0 .
$$

This completes the verification of $(5.6)$, so 5.2 is proven. 


\section{Proofs of the results of Section 3}

In the proofs, we will often use the relations

$$
\max _{1 \leq i \leq p}\left|\hat{\lambda}_{i}-\lambda_{i}\right| \stackrel{P}{\rightarrow} 0, \text { and } \max _{1 \leq i \leq p} \| \hat{\varphi}_{i}-\hat{c}_{i} \varphi_{i}|| \stackrel{P}{\rightarrow} 0 \quad \text { as } \min (M, N) \rightarrow \infty,
$$

where $\hat{c}_{i}=\operatorname{sign}\left(\left\langle\hat{\varphi}_{i}, \varphi_{i}\right\rangle\right)$, Analogous relations have been extensively used for the eigenvalues and the eigenfunctions of the empirical and population covariance operators, see Bosq (2000), Gabrys and Kokoszka (2007), Horváth et al. (2010), Panaretos et al. (2010), among many others, but they hold in much greater generality, see Chapter 2 of Horváth and Kokoszka (2011). Under 3.13), they hold for the eigenelements of the operators $\hat{D}$ and $D$ defined in Section 3 .

Proof of Theorem 3.1; By Theorem 2.1, assumptions (1.1)-(1.2) imply that, as $N \rightarrow$ $\infty$ and $M \rightarrow \infty$

$$
\left(N^{-1 / 2} \sum_{1 \leq i \leq N} \varepsilon_{i}, M^{-1 / 2} \sum_{1 \leq j \leq N} \varepsilon_{j}^{*}\right) \stackrel{d}{\rightarrow}\left(\Gamma^{(1)}, \Gamma^{(2)}\right),
$$

where $\Gamma^{(1)}$ and $\Gamma^{(2)}$ are independent $L^{2}$-valued mean zero Gaussian processes with covariances

$$
E\left[\Gamma^{(1)}(t) \Gamma^{(1)}(s)\right]=c(t, s), \quad E\left[\Gamma^{(2)}(t) \Gamma^{(2)}(s)\right]=c^{*}(t, s) .
$$

Hence

$$
\frac{N M}{N+M} \int\left(N^{-1} \sum_{1 \leq i \leq N} \varepsilon_{i}(t)-M^{-1} \sum_{1 \leq j \leq M} \varepsilon_{j}^{*}(t)\right)^{2} d t \stackrel{d}{\rightarrow} \int \Gamma(t) d t
$$

where $\Gamma(t)=(1-\theta)^{1 / 2} \Gamma^{(1)}(t)+\theta^{1 / 2} \Gamma^{(2)}(t)$. The conclusion of Theorem 3.1 now follows.

Proof of Theorem 3.2; By the ergodic theorem in a Hilbert space, $\left\|\bar{X}_{N}-\mu\right\|=o_{P}(1)$ and $\left\|\bar{X}_{M}-\mu^{*}\right\|=o_{P}(1)$, which imply the result.

Proof of Theorem 3.3. Under assumptions (2.2) and (1.2), we have, jointly,

$$
\left\{N^{-1 / 2} \sum_{1 \leq i \leq N}\left\langle\varepsilon_{i}, \varphi_{k}\right\rangle, 1 \leq k \leq p\right\} \stackrel{d}{\rightarrow} \mathbf{N}_{p}^{(1)}\left(\mathbf{0}, \mathbf{Q}^{(1)}\right)
$$

and

$$
\left\{M^{-1 / 2} \sum_{1 \leq j \leq M}\left\langle\varepsilon_{j}^{*}, \varphi_{k}\right\rangle, 1 \leq k \leq p\right\} \stackrel{d}{\rightarrow} \mathbf{N}_{p}^{(2)}\left(\mathbf{0}, \mathbf{Q}^{(2)}\right)
$$


where $\mathbf{N}_{p}^{(1)}\left(\mathbf{0}, \mathbf{Q}^{(1)}\right)$ and $\mathbf{N}_{p}^{(2)}\left(\mathbf{0}, \mathbf{Q}^{(2)}\right)$ are independent $p$-dimensional normal random vectors. Since

$$
a_{k}=N^{-1} \sum_{1 \leq i \leq N}\left\langle\varepsilon_{i}, \varphi_{k}\right\rangle-M^{-1} \sum_{1 \leq j \leq M}\left\langle\varepsilon_{j}^{*}, \varphi_{k}\right\rangle . \quad 1 \leq i \leq p
$$

3.10 holds with $\mathbf{Q}=(1-\theta) \mathbf{Q}^{(1)}+\theta \mathbf{Q}^{(2)}$.

Next we observe that the matrix $\mathbf{Q}=(Q(k, \ell), 1 \leq k, \ell \leq p)$ satisfies

$$
Q(k, \ell)=\iint d(t, s) \varphi_{k}(t) \varphi_{\ell}(s) d t d s=\lambda_{k} \delta_{k \ell}
$$

where $\delta_{i j}$ is Kronecker's delta, using the fact that the $\varphi_{i}$ are orthonormal eigenfunctions and the $\lambda_{i}$ are the corresponding eigenvalues.

Recall now relations (6.1), and observe that

$$
\begin{aligned}
\hat{a}_{k}=\hat{c}_{k}\left(N^{-1} \sum_{1 \leq i \leq N}\left\langle\varepsilon_{i}, \varphi_{k}\right\rangle-M^{-1} \sum_{1 \leq j \leq M}\left\langle\varepsilon_{j}^{*}, \varphi_{k}\right\rangle\right) \\
+\left\langle N^{-1} \sum_{1 \leq i \leq N} \varepsilon_{i}-M^{-1} \sum_{1 \leq j \leq M} \varepsilon_{j}^{*}, \hat{\varphi}_{k}-\hat{c}_{k} \varphi_{k}\right\rangle .
\end{aligned}
$$

By 6.1 and 6.2,

$$
\begin{aligned}
\left(\frac{N M}{N+M}\right)^{1 / 2} & \left|\left\langle\frac{1}{N} \sum_{1 \leq i \leq N} \varepsilon_{i}-\frac{1}{M} \sum_{1 \leq j \leq M} \varepsilon_{j}^{*}, \hat{\varphi}_{k}-\hat{c}_{k} \varphi_{k}\right\rangle\right| \\
& \leq U_{N, M}^{1 / 2}\left(\int\left(\hat{\varphi}_{k}-\hat{c}_{k} \varphi_{k}\right)^{2} d t\right)^{1 / 2} \\
& =o_{P}(1) .
\end{aligned}
$$

Hence the result follows immediately from 3.10 and the diagonality of $\mathbf{Q}$.

Proof OF TheOrem 3.4; By the ergodic theorem $a_{i} \stackrel{P}{\rightarrow}\left\langle\mu-\mu^{*}, \varphi_{i}\right\rangle, 1 \leq i \leq p$. Since relations 6.1 hold also under $H_{A}$, the result is proven.

Acknowledgements. The research was partially supported by NSF grants DMS-0905400 and DMS-0931948. 


\section{References}

Anderson, T. W. (1971). The Statistical Analysis of Time Series. Wiley and Sons.

Andrews, D. W. K. (1991). Heteroskedasticity and autocorrelation consistent covariance matrix estimation. Econometrica, 59, 817-858.

Andrews, D. W. K. and Monahan, J. C. (1992). An improved heteroskedasticity and autocorrelation consistent covariance matrix estimator. Econometrica, 60, 953-966.

Bosq, D. (2000). Linear Processes in Function Spaces. Springer, New York.

Brockwell, P. J. and Davis, R. A. (1991). Time Series: Theory and Methods. Springer, New York.

Doukhan, P. and Louhichi, S. (1999). A new weak dependence and applications to moment inequalities. Stochastic Processes and their Applications, 84, 313-343.

Gabrys, R., Horváth, L. and Kokoszka, P. (2010). Tests for error correlation in the functional linear model. Journal of the American Statistical Association, 105, 1113-1125.

Gabrys, R. and Kokoszka, P. (2007). Portmanteau test of independence for functional observations. Journal of the American Statistical Association, 102, 1338-1348.

Hamilton, J. D. (1994). Time Series Analysis. Princeton University Press, Princeton, NJ.

Hörmann, S. and Kokoszka, P. (2010). Weakly dependent functional data. The Annals of Statistics, 38, 1845-1884.

Horváth, L., Hušková, M. and Kokoszka, P. (2010). Testing the stability of the functional autoregressive process. Journal of Multivariate Analysis, 101, 352-367.

Horváth, L. and Kokoszka, P. (2011). Inference for Functional Data with Applications. Springer Series in Statistics. Springer. Forthcoming.

Horváth, L., Kokoszka, P. and Reimherr, M. (2009). Two sample inference in functional linear models. Canadian Journal of Statistics, 37, 571-591.

Kargin, V. and Onatski, A. (2008). Curve forecasting by functional autoregression. Journal of Multivariate Analysis, 99, 2508-2526.

Müller, H-G. and Stadtmüller, U. (2005). Generalized functional linear models. The Annals of Statistics, 33, 774-805.

Panaretos, V. M., Kraus, D. and Maddocks, J. H. (2010). Second-order comparison of gaussian random functions and the geometry of DNA minicircles. Journal of the American Statistical Association, 000, 000-000; Forthcoming.

Ramsay, J. O. and Silverman, B. W. (2005). Functional Data Analysis. Springer.

Wu, W. (2005). Nonlinear System Theory: Another Look at Dependence. Proceedings of The National Academy of Sciences of the United States, volume 102. National Academy of Sciences.

$\mathrm{Wu}, \mathrm{W}$. (2007). Strong invariance principles for dependent random variables. The Annals of Probability, 35, 2294-2320. 